\title{
Globalisierung von Forschung und Entwicklung der weltweit forschungsstärksten Unternehmen
}

\author{
Mit Patentdaten der weltweit forschungsstärksten Unternehmen lassen sich Informationen \\ über die regionale und technologische Verteilung ihrer Forschungsaktivitäten generieren. \\ Daraus ergeben sich Anhaltspunkte zu den Motiven der Internationalisierung. Unternehmen \\ betreiben Forschung und Entwicklung im Ausland überwiegend in technologischen \\ Feldern, wo sie bereits stark sind und der ausländische Standort über Vorteile verfügt. Die \\ wichtigsten Heimatländer forschender Unternehmen sind auch die wichtigsten Zielländer \\ der Auslandsforschung. Somit dürften nicht nur die Unternehmen, sondern auch diese \\ Forschungsstandorte von der Internationalisierung der Forschung und Entwicklung profitieren.
}

Die meisten forschungsstarken multinationalen Unternehmen betreiben auch Forschung und Entwicklung (FuE) im Ausland, oft an mehreren Standorten. Allerdings gibt es nur für wenige Heimatländer dieser Unternehmen Informationen über den Umfang, die Zielregionen, die Branchen- und Technologieschwerpunkte dieser internationalen Aktivitäten. Mit Patentdaten der rund 1.700 weltweit forschungsstärksten Unternehmen kann diese Informationslücke zumindest für deren patentrelevante Forschung geschlossen werden. Die Daten offenbaren, wo die Erfinder:innen dieser Unternehmen wohnen und welche technologischen Schwerpunkte sie im Heimat- und Ausland bearbeiten. Auf dieser Grundlage lässt sich für die forschungsstärksten Unternehmen der Internationalisierungsgrad und die weltweite regionale Verteilung ihrer FuE ermitteln. Kombiniert mit den technologischen Schwerpunkten der patentrelevanten FuE-Tätigkeit können daraus Anhaltspunkte zu den Motiven der Internationalisierung gewonnen werden.

Wenn Unternehmen FuE im Ausland durchführen, wird dies gelegentlich als Verlust für den heimischen Standort interpretiert. ${ }^{1}$ Dabei ist es für Unternehmen oft notwendig, ihre Produkte und Prozesse an die Bedürfnisse ausländischer Kunden anzupassen und dabei die lokalen Bedingun-

(C) Der/die Autor:in(nen) 2021. Open Access: Dieser Artikel wird unter der Creative Commons Namensnennung 4.0 International Lizenz veröffentlicht (creativecommons.org/licenses/by/4.0/deed.de).

Open Access wird durch die ZBW - Leibniz-Informationszentrum Wirtschaft gefördert.

1 Bofinger beschreibt Beispiele des Aufbaus anwendungsorientierter Forschungseinrichtungen deutscher Unternehmen wie Siemens, Bosch und Schaeffler in China als Verlagerung. Er sieht darin eine Bestätigung der These, wonach Unternehmen ihre FuE-Aktivitäten in Länder verlagern, die hierfür bessere FuE-Infrastrukturen und günstigere finanzielle Förderung vorweisen können (SVR, 2018, 79). gen zu berücksichtigen. Sie müssen deshalb auch in den wichtigsten Zielmärkten forschen. Der Aufbau von eigenen Forschungslaboren im Ausland dient aber auch dazu, dort neues technologisches Wissen bei Wettbewerbern, Hochschulen und Forschungsinstituten zu erwerben. Schließlich ist auch der Zugriff auf qualifiziertes Forschungspersonal und sein spezielles Know-how ein wichtiges Motiv für FuE im Ausland, besonders wenn solches Personal im Heimatland knapp ist (OECD, 2008).

\section{FuE-Aufwendungen von Unternehmen im Ausland}

Nur in wenigen Ländern, darunter den USA, Deutschland und Schweden, liegen nationale Statistiken zu den FuEAufwendungen der einheimischen Unternehmen im In- und Ausland vor (Gehrke et al., 2020). Daraus lässt sich der Internationalisierungsgrad der FuE dieser Unternehmen als Anteil der FuE-Aufwendungen im Ausland an den globalen FuE-Aufwendungen berechnen. In deutschen Unternehmen erhöhte sich der Auslandsanteil von 1997 bis 2017 von $23 \%$ auf $38 \%$ (vgl. Abbildung 1). Allerdings ging er zwischenzeitlich zurück und stieg nach 2007 erneut. Schwedische Unternehmen steigerten den Auslandsanteil im

Dr. Heike Belitz ist wissenschaftliche Mitarbeiterin in der Abteilung Unternehmen und Märkte am DIW Berlin.

Prof. Dr. Anna Lejpras lehrt Allgemeine Betriebswirtschaftslehre an der BSP Business School Berlin. 


\section{Abbildung 1}

Anteil der FuE-Unternehmensaufwendungen im Ausland

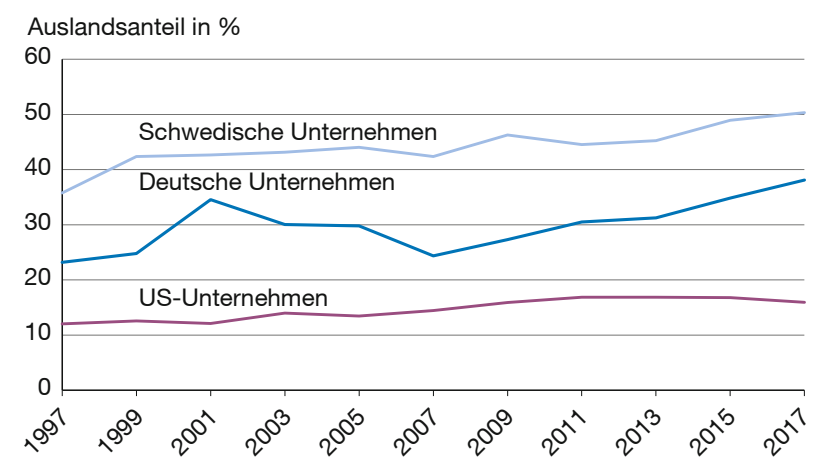

Quelle: nationale Statistiken; eigene Berechnungen.

gleichen Zeitraum von $36 \%$ auf $50 \%$. In US-Unternehmen stieg dieser Anteil von $12 \%$ (1997) auf $16 \%$ (2010), stagnierte jedoch danach. Insgesamt nahm die Internationalisierung der FuE in diesen Unternehmen moderat zu.

Um einen tieferen Einblick in das Ausmaß sowie die regionalen und technologischen Schwerpunkte der Auslandsforschung großer Unternehmen zu bekommen, können Informationen aus Patentanmeldungen von weltweit knapp 1.700 besonders forschungsstarken Unternehmen von 2012 bis 2014 genutzt werden. Dazu wurden zwei Datensätze verbunden: Ein Datensatz mit Informationen zu den FuE-Aufwendungen und Patentanmeldungen der 2.000 weltweit forschungsstärksten Unternehmen von 2012 bis 2014 (ECJRC/OECD COR\&DIPC) database, v.1. 2017 des EC-JRC Institute for Prospective Technological Studies (IPTS) und des OECD Directorate for Science, Technology and Innovation) und die Patentdatenbank des Europäischen Patentamtes (EPO) mit bibliografischen Daten zu Patenten (EPO Worldwide Patent Statistical Database PATSTAT, Frühling 2018). ${ }^{2}$ Um Doppelzählungen bei Mehrfachanmeldungen von Erfindungen bei mehreren Patentämtern zu vermeiden, wird die Auswertung auf Ebene der sogenannten Patentfamilien vorgenommen. Patentfamilien fassen die verschiedenen Patentanmeldungen einer Erfindung an den fünf weltweit größten Patentämtern in Europa, den USA, China, Japan und Korea zusammen. Die technologische Ausrichtung der hinter den Erfindungen stehenden FuE-Aktivitäten wird anhand von 35 Technologiefeldern in fünf Bereichen abgebildet (Schmoch, 2008). Orte der Erfindung werden für eine Patentfamilie über die Wohnorte der Erfinder:innen ermittelt. Da eine Erfindung, abgebildet in einer Patentfamilie, unter Umständen mehreren Erfindern an verschiedenen Orten, mehreren Patenten,

2 Die Autorinnen danken Maximilian Priem für die Unterstützung beim Matching der beiden Datenbanken. mehreren Anmeldeunternehmen und mehreren Technologiefeldern zugeordnet werden kann, werden die Patentfamilien mit einer fraktionierten Zählweise gewichtet.

Wie Abbildung 1 zeigt, hat sich der Anteil der internationalen FuE-Aktivitäten der multinationalen Unternehmen aus den USA, Deutschland und Schweden seit 2014 nur wenig verändert, sodass mit den Daten der 1.668 weltweit forschungsstärksten Unternehmen für 2012 bis 2014 ein immer noch aktuelles Bild der regionalen und technologischen Verteilung ihrer FuE-Aktivitäten gezeichnet werden kann. ${ }^{3}$ Die gesamten FuE-Aufwendungen dieser Unternehmen lagen 2014 bei 571 Mrd. Euro. Das waren fast drei Viertel aller privaten FuE-Aufwendungen an den weltweit wichtigsten Forschungsstandorten zusammen (USA, EU28, China, Japan, Südkorea, Schweiz, Kanada, Taiwan, Norwegen) in Höhe von etwa 770 Mrd. Euro.

US-Unternehmen haben mit gut $38 \%$ den größten Anteil an den FuE-Aufwendungen des Samples (vgl. Abbildung 2). Mit $23 \%$ deutlich geringer ist inr Anteil an den Patentgewichten. Umgekehrt ist es bei den zweitplatzierten japanischen Unternehmen mit nur gut $15 \%$ Anteil an den FuE-Aufwendungen, aber $34 \%$ Anteil an den Patentgewichten des Samples. Bei den FuE-Aufwendungen folgen die deutschen Unternehmen mit einem Anteil von fast $11 \%$ auf dem dritten Platz, bei den Patentgewichten sind es jedoch die südkoreanischen Unternehmen. Sowohl japanische als auch südkoreanische Unternehmen weisen also deutlich weniger FuE-Aufwendungen je Patent auf als die US-amerikanischen, die deutschen und anderen europäischen Unternehmen. ${ }^{4}$ In den Unternehmen aus den führenden acht Heimatländern, zu denen neben den genannten auch Frankreich, die Schweiz, Großbritannien und China gehören, konzentrieren sich jedoch rund $87 \%$ sowohl der FuE-Investitionen als auch der Patente des untersuchten Unternehmenssamples.

\section{Differenzierung nach Heimat- und Zielländern}

Immerhin 363 FuE-Investierende, also gut ein Fünftel haben keine patentrelevante Forschung im Ausland, sie forschen nur in ihrem jeweiligen Heimatland. Im Durchschnitt liegt der Anteil der gewichteten Patente im Ausland in allen untersuchten Unternehmen bei $21 \%$, in den international forschenden Unternehmen aber bereits bei $34 \%$. Die höchsten FuE-Anteile im Ausland gemessen an den Patentgewichten haben Unternehmen einiger relativ kleiner Heimatländer, wie

3 Die FuE-Aktivitäten deutscher Unternehmen im Ausland wurden mit dem beschriebenen Datensatz in Belitz et al. (2019) untersucht.

4 Dies hängt sowohl mit der unterschiedlichen Patentierungsneigung in verschiedenen Unternehmen, Ländern, Branchen und Technologiefeldern zusammen als auch mit unterschiedlicher Patentproduktivität (Patente je Einheit FuE-Aufwand). 
Abbildung 2

Anteile der weltweiten FuE-Aufwendungen und Patentgewichte nach Heimatländern

FuE-Aufwand 2012 bis 2014 nach Heimatländern

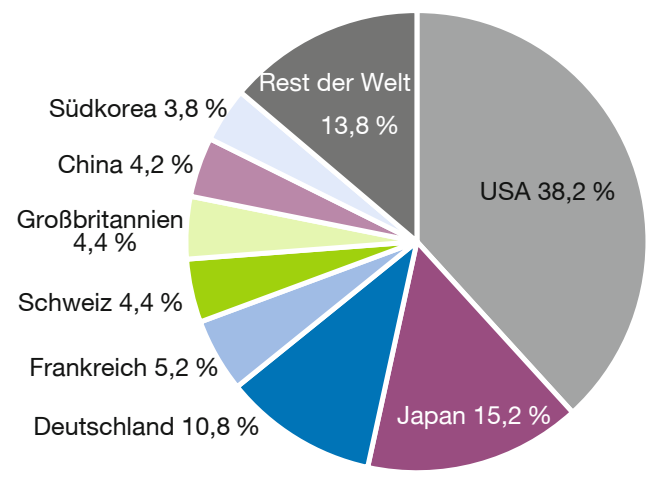

Patentgewichte 2012 bis 2014 nach Heimatländern

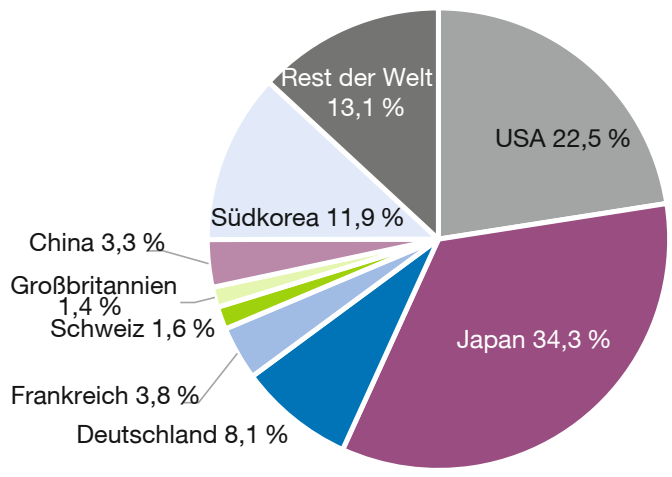

Quellen: IPTS und OECD Directorate for Science, Technology and Innovation; EPO; eigene Berechnungen.

Irland, Schweiz und Niederlande (vgl. Tabelle 1). Sehr geringe Auslandsanteile haben Unternehmen aus Japan, China und Südkorea. Unter den Unternehmen wichtiger Heimatländer außerhalb Asiens haben deutsche Unternehmen den geringsten Auslandsanteil. Im Vergleich zu den Unternehmen aus den USA, Großbritannien und Frankreich ist ihre FuE unterdurchschnittlich internationalisiert. Dies gilt auch, wenn man die Internationalisierung der Forschung über Kontinente betrachtet (vgl. Tabelle 1). Der mittlere Anteil der gewichteten Patente von einem anderen Kontinent, etwa bei EU-Unternehmen außerhalb Europas, liegt bezogen auf das gesamte Sample bei knapp $14 \%$. Beim Internationalisierungsgrad nach Kontinenten ist die Varianz zwischen den Unternehmen deutlich geringer als die des Internationalisierungsgrades nach Ländern. D. h., bezogen auf den Anteil interkontinentaler FuE, sind sich die Unternehmen ähnlicher.

Von der insgesamt im Ausland durchgeführten FuE entfällt der größte Anteil auf europäische Unternehmen, die in anderen EU-Ländern forschen (24\%) (vgl. Tabelle 2). Es folgen nordamerikanische (überwiegend US-amerikanische)
Tabelle 1

Internationalisierungsgrad der Unternehmen großer Heimatländer

\begin{tabular}{|c|c|c|c|c|c|c|}
\hline & $\begin{array}{l}\text { Zahl } \\
\text { der } \\
\text { Unter- } \\
\text { neh- } \\
\text { men }\end{array}$ & Patentg & $\begin{array}{l}\text { gewichte } \\
\text { Dar- } \\
\text { unter: } \\
\text { Ausland }\end{array}$ & Rang & $\begin{array}{l}\text { Anteil } \\
\text { Ausland } \\
\text { in \% }\end{array}$ & $\begin{array}{l}\text { Anteil } \\
\text { anderer } \\
\text { Konti- } \\
\text { nente in \% }\end{array}$ \\
\hline Japan & 292 & 114.858 & 6.789 & 3 & 5,9 & 5,0 \\
\hline USA & 570 & 75.533 & 22.266 & 1 & 29,5 & 27,6 \\
\hline Südkorea & 57 & 39.926 & 2.788 & 8 & 7,0 & 3,7 \\
\hline Deutschland & 104 & 27.121 & 7.221 & 2 & 26,6 & 13,8 \\
\hline Taiwan & 74 & 18.936 & 4.334 & 6 & 22,9 & 2,9 \\
\hline Frankreich & 65 & 12.618 & 4.376 & 5 & 34,7 & 16,0 \\
\hline China & 107 & 11.129 & 959 & 13 & 8,6 & 7,4 \\
\hline Niederlande & 32 & 7.607 & 5.466 & 4 & 71,9 & 19,2 \\
\hline Schweiz & 48 & 5.328 & 3.848 & 7 & 72,2 & 29,4 \\
\hline Großbritannien & 89 & 4.728 & 2.706 & 9 & 57,2 & 36,1 \\
\hline Schweden & 26 & 4.656 & 2.700 & 10 & 58,0 & 22,9 \\
\hline Irland & 15 & 2.597 & 2.562 & 11 & 98,6 & 86,8 \\
\hline Finnland & 18 & 2.466 & 1.051 & 12 & 42,6 & 23,5 \\
\hline Kanada & 19 & 1.357 & 556 & 14 & 41,0 & 22,3 \\
\hline Dänemark & 19 & 999 & 348 & 15 & 34,9 & 20,6 \\
\hline
\end{tabular}

Quellen: IPTS und OECD Directorate for Science, Technology and Innovation; EPO; eigene Berechnungen.

Unternehmen mit Auslandsforschung in Europa und europäische Unternehmen mit FuE in Nordamerika (je rund $16 \%)$. Die „Bilanz“ der gegenseitigen Verflechtung der Unternehmensforschung dieser beiden Kontinente ist also etwa ausgeglichen. Nordamerikanische Unternehmen führen auch viel FuE in Asien durch (11\%) und asiatische Unternehmen in jeweils anderen asiatischen Ländern (9\%). Während Europa und Asien als Heimatregion und als Zielregion von Unternehmensforschung im Ausland etwa den gleichen Anteil haben, ist Nordamerika stärker Heimat- als Zielregion von Auslandsforschung. Nordamerikanische und vor allem die US-Unternehmen melden etwas mehr Patente auf Basis von FuE im Ausland an als umgekehrt ausländische Unternehmen auf Basis von FuE in den USA. ${ }^{5}$

Unter den großen Heimatländern forschungsstarker Unternehmen haben Deutschland und Großbritannien mehr FuE aus dem Ausland angezogen als ihre Unternehmen im Ausland durchführen (vgl. Abbildung 3). In den USA, Japan, den Niederlanden, Frankreich und der Schweiz ist es umgekehrt. Die Unternehmen dieser Länder betreiben im

5 Die FuE-Aufwendungen von US-Tochterunternehmen im Mehrheitsbesitz im Ausland (55 Mrd. US-\$) und ausländischer Tochterunternehmen im Mehrheitsbesitz in den USA (57 Mrd. US-\$) waren jedoch 2014 etwa ausgeglichen (U.S. Bureau of Economic Analysis, o. D.). 
Tabelle 2

Verteilung der Auslandsforschung nach Kontinenten in $\%$

\begin{tabular}{lcrrrr} 
& \multicolumn{2}{c}{ Zielkontinent } & & \\
Heimatkontinent & Europa & Asien & Nordamerika & Rest & Insgesamt \\
\hline Europa & 23,7 & 4,7 & 15,6 & 0,9 & 44,8 \\
\hline Asien & 4,8 & 9,0 & 7,4 & 0,8 & 22,0 \\
\hline Nordamerika & 16,6 & 10,6 & 2,4 & 2,7 & 32,3 \\
\hline Restliche Welt & 0,3 & 0,1 & 0,4 & 0,0 & 0,9 \\
\hline Insgesamt & 45,4 & 24,4 & 25,8 & 4,4 & 100,0
\end{tabular}

Quellen: IPTS und OECD Directorate for Science, Technology and Innovation; EPO; eigene Berechnungen.

Ausland mehr patentrelevante FuE als ausländische Unternehmen im Inland. Europäische Unternehmen konzentrieren ihre Auslandsforschung in Europa, an zweiter Stelle folgt Nordamerika (vgl. Abbildung 4). Nur in britischen Unternehmen ist es umgekehrt. Asiatische Unternehmen forschen - bei relativ geringem Internationalisierungsgrad - vor allem auf dem asiatischen Kontinent und in Nordamerika. Nur die japanischen Unternehmen setzen auch in Europa einen Schwerpunkt. Die US-amerikanischen Unternehmen forschen zu deutlich höheren Anteilen in Asien als die europäischen Unternehmen. Für die US-Unternehmen ist zudem Israel ein wichtiger Forschungsstandort.

\section{Internationale Forschung}

Der wichtigste Technologiebereich, in dem die forschungsstarken Unternehmen Patente anmelden, ist die Elektrotechnik, auf die insgesamt und im Ausland rund $45 \%$ der Patentgewichte entfallen (vgl. Tabelle 3). Die größten Tech-

\section{Abbildung 4}

Verteilung der Auslandsforschung der Unternehmen ausgewählter Heimatländer auf Zielregionen

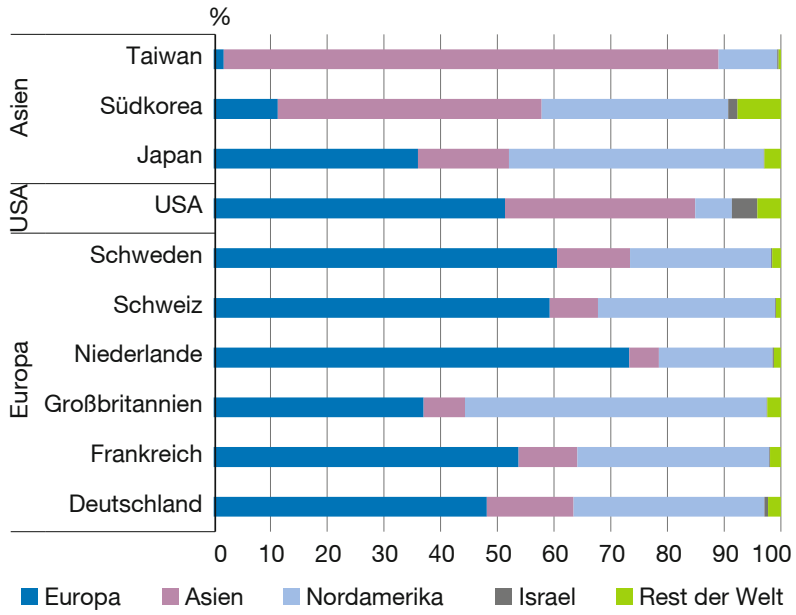

Quellen: IPTS und OECD Directorate for Science, Technology and Innovation; EPO; eigene Berechnungen.
Abbildung 3

Patentgewichte einheimischer Unternehmen im Ausland und ausländischer Unternehmen im Inland

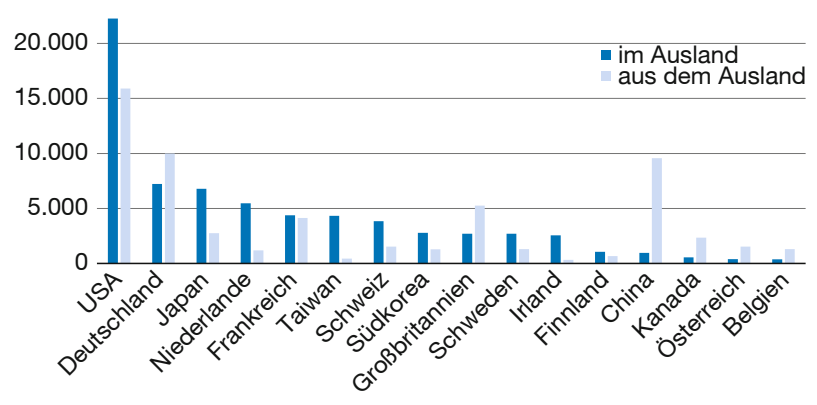

Quellen: IPTS und OECD Directorate for Science, Technology and Innovation; EPO; eigene Berechnungen.

nologiefelder sind dabei die Computertechnik, elektrische Maschinen und Anlagen sowie die digitale Kommunikationstechnik (vgl. Abbildung 5). Sowohl zwischen den Technologiebereichen als auch zwischen den Technologiefeldern variieren die Auslandsanteile der Patente kaum. Die höchsten Internationalisierungsgrade haben die Technologiefelder im Bereich Chemie, darunter Pharmazie (37\%), Nahrungsmittelchemie, Biotechnologie und Medizintechnik (31\%). Auch im Bauwesen wird mit knapp $35 \%$ ein vergleichsweise hoher Internationalisierungsgrad erreicht. Die Internationalisierung variiert zwischen den Technologiefeldern aber deutlich weniger als die zwischen den Heimatländern der Unternehmen.Zur Charakterisierung der Motive für die Internationalisierung von FuE wird ein Klassifizierungsschema verwendet, das bereits mehrfach angewendet wurde (Patel und Vega, 1999; Le Bas und Sierra, 2002; Laurens et al., 2015). Für jede Technologie werden vier Internationalisierungsstrategien danach unterschieden, ob ein Unternehmen im Heimatland auf die Technologie spezialisiert ist und ob das Zielland auf diese Technologie spezialisiert ist. Dazu werden zwei technologische Spezialisierungsmaße auf Basis des RTA (Revealed Technological Advantage) berechnet ${ }^{6}$ :

- Um die technologische Spezialisierung eines Unternehmens im Heimatland (RTA Heimat) zu bestimmen, werden zwei Anteile in Relation gesetzt: Erstens der Anteil der von einem Unternehmen in einem Technologiefeld erforschten Patentanmeldungen an allen Patentanmeldungen des Unternehmens im Heimatland. Zweitens der Anteil der in diesem Technologiefeld von allen Unternehmen angemeldeten Patente an allen Patenten weltweit.

6 Der RTA wurde zunächst zur Messung der internationalen Handelsspezialisierung entwickelt, aber auch zur Erfassung der technologischen Spezialisierung mit Patentdaten genutzt. Er misst die relative Konzentration der Erfindertätigkeit (Patentfamilien p) auf bestimmte Technologien im Vergleich zu einer Grundgesamtheit von Unternehmen. RTA $\left(p_{t} / \sum_{t} p_{t r}\right) /\left(\sum_{t} p_{t r} / \sum_{t r} p_{t r}\right)$. Dabei steht $t$ für den Index des Technologiefelds und $r$ für den Index der jeweiligen Unternehmensauswahl. 
Tabelle 3

Patentgewichte forschungsstarker Unternehmen großer Heimatländer nach Technologiebereichen

\begin{tabular}{lrrrrcc} 
Technologiebereich & Gesamt & in \% & Ausland & in \% & Anteil Ausland in \% \\
\hline Elektrotechnik & 160.085 & 47,8 & 31.754 & 44,9 & 19,8 \\
\hline Instrumente & 53.813 & 16,1 & 10.878 & 15,4 & 20,2 \\
\hline Chemie & 39.690 & 11,8 & 9.829 & 13,9 & 24,8 \\
\hline Maschinenbau & 71.148 & 21,2 & 15.316 & 21,7 & 21,5 \\
\hline Andere & 10.473 & 3,1 & 2.962 & 4,2 & 28,3 \\
\hline Insgesamt & 335.212 & 100,0 & 70.741 & 100,0 & 21,1
\end{tabular}

Quellen: IPTS und OECD Directorate for Science, Technology and Innovation; EPO; eigene Berechnungen.

- Um die technologische Spezialisierung des Ziellandes (RTA Zielland) zu bestimmen, werden zwei Anteile in Relation gesetzt: Erstens der Anteil der Patentanmeldungen aller dort forschenden Unternehmen in einem bestimmten Technologiefeld an allen Patentanmeldungen in dem Zielland. Zweitens der Anteil der weltweit in diesem Technologiefeld angemeldeten Patente an allen Patenten.

Je nach Ausprägung der Spezialisierungsmaße gibt es vier Internationalisierungsstrategien für ein Technologiefeld in einer ausländischen Zielregion (vgl. Tabelle 4):

1. In der wissenserweiternden bzw. -ergänzenden Strategie (WE) ist das Unternehmen im jeweiligen Technologiefeld in der Heimat stark, und auch das Zielland verfügt über Vorteile. Das Unternehmen nutzt die komplementäre Stärke im Ausland zur Erweiterung und Ergänzung der in der Heimat aufgebauten technologischen Stärke.

2. In der wissensnutzenden Strategie (WN) ist die Forschung im Zielland relativ schwach und das Unternehmen nutzt dort die in der Heimat erarbeiteten technologischen Vorteile. Damit wird im Ausland eher technische Unterstützung für den Absatz und die dortige Produktion des eigenen Unternehmens geleistet.

3. Mit einer technologiesuchenden Strategie (TS) versucht das Unternehmen, seine technologische Schwäche am Heimatstandort durch Forschung in den Ländern auszugleichen, die in diesen Feldern stark sind. So kann innen Forschung an Spitzenstandorten den Zugang zu neuen Hochtechnologien ermöglichen.

4. In der marktorientierten Strategie (MO) spielen technologische Motive keine wichtige Rolle, da weder das Heimat- noch das Zielland auf das jeweilige Forschungsfeld spezialisiert sind. Die Forschung im Ausland könnte etwa Ergebnis einer Unternehmensübernahme sein, bei der nichttechnologische Gründe entscheidend waren.
Abbildung 5

Patentgewichte nach Technologiefeldern im Heimatund Ausland

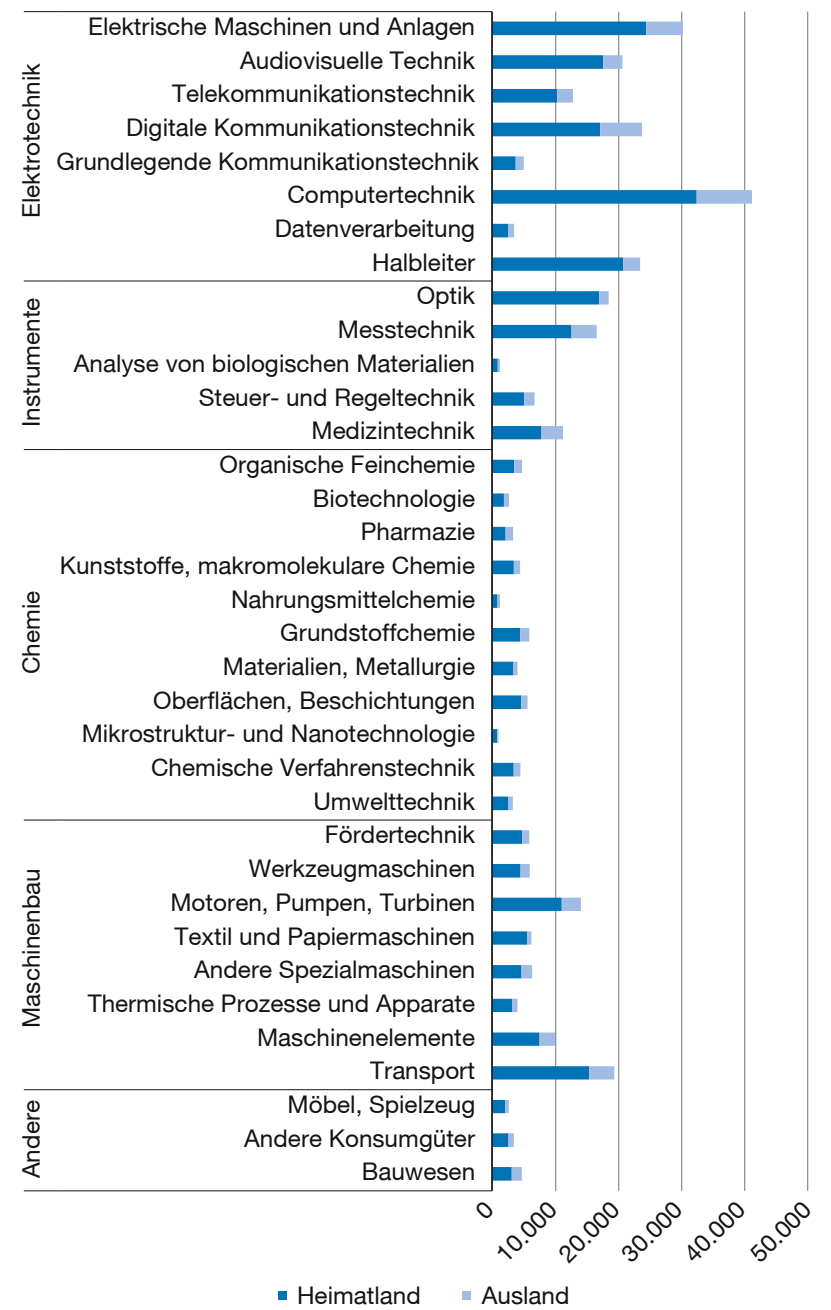

Quellen: IPTS und OECD Directorate for Science, Technology and Innovation; EPO; eigene Berechnungen.

Im Ausland betreiben Unternehmen FuE überwiegend aus einer Position der technologischen Stärke im Heimatland. $82 \%$ der Patente der forschungsstarken Unternehmen werden in Technologiefeldern angemeldet, auf die diese Unternehmen im Heimatland spezialisiert sind. Darunter entfallen die meisten Patente (55\%) auf Technologiefelder, auf die auch die Forschungsstandorte im Ausland spezialisiert sind (vgl. Tabelle 5). Auf eine technologiesuchende Strategie, bei der das Unternehmen im Ausland in Feldern forscht, in denen es im Heimatland nicht über Vorteile verfügt, weisen nur $9 \%$ der Auslandspatente hin. Der Erwerb von technologischem Wissen im Ausland wird von den Unternehmen also vor allem dann angestrebt, wenn bereits eine technologische Stärke am Heimatstandort besteht und nur deutlich seltener aus einer Position der Schwäche heraus. Dies trifft für Unternehmen aus allen Ländern zu und ist für Unterneh- 
Tabelle 4

Internationalisierungsstrategien der Forschung

\begin{tabular}{|c|c|c|c|}
\hline \multirow{2}{*}{\multicolumn{2}{|c|}{$\begin{array}{l}\text { Technologische } \\
\text { Spezialisierung ... }\end{array}$}} & \multicolumn{2}{|c|}{... der privaten Forschung im Zielland } \\
\hline & & stark & schwach \\
\hline $\begin{array}{l}\ldots \text { des } \\
\text { Unterneh- }\end{array}$ & stark & $\begin{array}{l}\text { Wissensergänzend (WE) } \\
\text { RTA Heimat>1 } \\
\text { RTA Ziel>1 }\end{array}$ & $\begin{array}{l}\text { Wissensnutzend (WN) } \\
\text { RTA Heimat }>1 \\
\text { RTA Ziel }<1\end{array}$ \\
\hline $\begin{array}{l}\text { Heimat- } \\
\text { land }\end{array}$ & schwach & $\begin{array}{l}\text { Technologiesuchend (TS) } \\
\text { RTA Heimat<1 } \\
\text { RTA Ziel }>1\end{array}$ & $\begin{array}{l}\text { Marktorientiert (MO) } \\
\text { RTA Heimat<1 } \\
\text { RTA Zie<1 }\end{array}$ \\
\hline
\end{tabular}

Quelle: Patel und Vega (1999).

men aus dem Entwicklungsland Indien sogar besonders ausgeprägt. Betrachtet man die Verteilung der Internationalisierungsstrategien aus der Sicht der Zielländer der Auslandsforschung, dann ergibt sich ein ähnliches Bild der Verteilung der Internationalisierungsmotive (vgl. Tabelle 6). Das verwundert nicht, denn die wichtigsten Heimatländer international forschender Unternehmen sind auch die wichtigsten Zielländer ihrer Auslandsforschung (vgl. Abbildung 3).

Im für die internationale Forschung besonders bedeutenden Bereich der Elektrotechnik haben wissensnutzende Internationalisierungsmotive eine leicht überdurchschnittliche Bedeutung (vgl. Tabelle 7). Unternehmen forschen in den Zielländern häufiger, obwohl diese nicht auf die Technologien in diesem Bereich spezialisiert sind. Dies weist auf die stärkere Nutzung heimischer Technologievorteile und die Anpassung an Marktbedingungen und Kundenwünsche in den Zielmärkten hin. Technologiesuchende Strate-

Tabelle 5

Anteile der Internationalisierungsstrategien der Unternehmen nach Heimatländern

Patentgewichte in \%

\begin{tabular}{llcrc} 
Heimatland & WE & WN & TS & MO \\
\hline USA & 55,5 & 26,3 & 9,2 & 9,0 \\
\hline Deutschland & 49,7 & 26,7 & 12,3 & 11,4 \\
\hline Japan & 43,0 & 32,2 & 15,6 & 9,2 \\
\hline Niederlande & 62,4 & 29,4 & 3,7 & 4,5 \\
\hline Frankreich & 55,2 & 25,9 & 11,0 & 7,8 \\
\hline Taiwan & 44,1 & 30,4 & 5,1 & 20,4 \\
\hline Schweiz & 59,3 & 21,1 & 10,5 & 9,2 \\
\hline Südkorea & 56,9 & 22,2 & 9,7 & 11,2 \\
\hline Großbritannien & 62,4 & 24,3 & 7,4 & 6,0 \\
\hline Schweden & 65,4 & 19,4 & 10,5 & 4,7 \\
\hline China & 58,4 & 26,1 & 8,2 & 7,3 \\
\hline Indien & 80,7 & 18,9 & 0,1 & 0,3 \\
\hline Insgesamt & 55,2 & 26,9 & 9,2 & 8,8 \\
\hline Quelen: & &
\end{tabular}

Quellen: IPTS und OECD Directorate for Science, Technology and Innovation; EPO; eigene Berechnungen.
Tabelle 6

Anteile der Internationalisierungsstrategien der Unternehmen in führenden Zielländern

Patentgewichte in \%

\begin{tabular}{lccrrr} 
& \multirow{2}{*}{$\begin{array}{c}\text { Nachrichtlich: aus } \\
\text { internationaler FuE }\end{array}$} & \multicolumn{4}{c}{ Internationalisierungsstrategien } \\
Zielland & 22,5 & 54,8 & 27,0 & 11,2 & 7,1 \\
\hline USA & 14,1 & 64,8 & 19,9 & 10,5 & 4,8 \\
\hline Deutschland & 13,5 & 53,9 & 27,4 & 4,2 & 14,5 \\
\hline China & 7,4 & 55,5 & 26,0 & 7,5 & 11,0 \\
\hline Großbritannien & 5,9 & 52,1 & 34,9 & 4,4 & 8,6 \\
\hline Frankreich & 3,9 & 33,6 & 42,8 & 12,8 & 10,7 \\
\hline Japan & 3,4 & 59,6 & 24,8 & 9,1 & 6,5 \\
\hline Indien & 3,3 & 56,0 & 28,3 & 7,7 & 7,9 \\
\hline Kanada & 3,1 & 60,1 & 24,3 & 10,3 & 5,3 \\
\hline Italien & 2,2 & 53,8 & 22,6 & 11,4 & 12,2 \\
\hline Österreich & 100,0 & 55,2 & 26,9 & 9,2 & 8,8 \\
\hline Gesamt & & & & &
\end{tabular}

Quellen: IPTS und OECD Directorate for Science, Technology and Innovation; EPO; eigene Berechnungen.

gien haben im Bereich der Instrumente einen leicht überdurchschnittlichen Anteil, insbesondere in der Messtechnik (20\%) sowie in der Steuer- und Regelungstechnik (17\%).

Die USA und Deutschland sind die beiden wichtigsten Heimat- und Zielländer der internationalen FuE der forschungsstarken multinationalen Unternehmen. Sie sind auch gegenseitig stark verflochten. Für deutsche Unternehmen sind die USA der wichtigste Forschungsstandort. Fast ein Drittel ihrer Patente im Ausland wurden dort erarbeitet. Auch für US-Unternehmen ist Deutschland ein wichtiger Forschungsstandort: Knapp $18 \%$ ihrer Patente im Ausland entfallen auf Deutschland. Insgesamt nutzen deutsche Unternehmen in den USA etwas weniger wissenserweiternde Strategien als umgekehrt US-Unternehmen in Deutschland (vgl. Tabelle 8). Technologiesuchende Strategien haben in deutschen Unternehmen in den USA ein etwas höheres Gewicht. Deutsche Unternehmen profitieren vor allem in der Computertechnik, aber auch in der Medizintechnik vom Vorsprung dort und erwerben neues Wissen durch Auslandsforschung (vgl. Abbildung 6). Auffällig ist die Ähnlichkeit der Schwerpunktsetzung der Unternehmen der beiden Länder im jeweils anderen Land in den beforschten Technologien. Die meisten FuE-Aktivitäten im Ausland entfallen sowohl in deutschen als auch in US-Unternehmen auf elektrische Maschinen und Anlagen, Transporttechnik, Computertechnik, Mess- und Medizintechnik sowie Motoren, Pumpen und Turbinen. Dabei forschen deutsche Unternehmen in den USA viel in den Technologien elektrische Maschinen und Anlagen sowie Transporttechnik, obwohl sie selbst, jedoch nicht die USA, darauf spezialisiert sind. Umgekehrt 
Tabelle 7

Patentgewichte der Internationalisierungsstrategien nach Technologiebereichen

Patentgewichte in \%

\begin{tabular}{lcccc} 
& \multicolumn{4}{c}{ Internationalisierungsstrategien } \\
& WE & WN & TS & MO \\
\hline Elektrotechnik & 47,0 & 33,4 & 9,1 & 10,5 \\
\hline Instrumente & 56,7 & 19,5 & 13,4 & 10,4 \\
\hline Chemie & 63,9 & 23,4 & 7,4 & 5,2 \\
\hline Maschinenbau & 62,9 & 22,4 & 7,9 & 6,8 \\
\hline Andere Bereiche & 69,0 & 19,1 & 6,5 & 5,4 \\
\hline Insgesamt & 55,2 & 26,9 & 9,2 & 8,8 \\
\hline
\end{tabular}

Quellen: IPTS und OECD Directorate for Science, Technology and Innovation; EPO; eigene Berechnungen.

forschen auch US-Unternehmen in Deutschland in großem Umfang in ihrem Kompetenzbereich Computertechnik und einigen anderen luK-Technologien, obwohl Deutschland hier keine technologischen Vorteile hat. In diesen Fällen dürften der Markt und die Anpassung an Kundenwünsche die dominierenden Motive der Auslandsforschung sein. In den anderen technologischen Schwerpunktfeldern der Auslandsforschung Mess-, Medizintechnik und Motoren, Pumpen und Turbinen verfügen beide Länder über Vorteile. In diesen Technologien ergänzen sich die Stärken der Heimat- und Zielstandorte, und die Unternehmen können vom Wissensaustausch profitieren. Technologieorientierte Motive der internationalen FuE wie Wissenserwerb durch Beobachtung und das Lernen von Wettbewerbern dürften hier gegenüber marktorientierten Motiven dominieren.

\section{Fazit}

Die höchsten FuE-Anteile im Ausland haben forschungsstarke Unternehmen einiger kleiner europäischer Länder, wie Irland, Schweiz und Niederlande. Große Unternehmen von dort müssen schon deshalb im Ausland forschen, da sie in der Heimat nicht das für sie erforderliche Technologieportfolio abdecken können. Deutlich geringer sind die Auslandsforschungsanteile asiatischer Unternehmen. Die Anteile interkontinentaler FuE sind zwischen den Unternehmen verschiedener Heimatländer ähnlicher. Deutsche Unternehmen sind im Vergleich zu Unternehmen aus den USA, Großbritannien und Frankreich weniger internationalisiert, auch bezüglich des FuE-Anteils in anderen Kontinenten. Dies deutet auf einen Nachholbedarf deutscher Unternehmen bei der Internationalisierung von Forschung verglichen mit Wettbewerbern hin. Während Europa und Asien als Heimat- und als Zielregion internationaler Unternehmensforschung etwa den gleichen Anteil haben, ist Nordamerika stärker Heimat- als Zielregion von patentrelevanter Auslandsforschung. Die „Bilanz" der gegenseitigen Verflechtung über Auslandsforschung zwischen Europa
Tabelle 8

Internationalisierungsstrategien der Unternehmen aus den USA und Deutschland

Patentgewichte in \%

Internationalisierungsstrategien

\begin{tabular}{llllr} 
& WE & WN & TS & MO \\
\hline US-Unternehmen in Deutschland & 63,8 & 20,6 & 10,7 & 4,8 \\
\hline Deutsche Unternehmen in den USA & 46,9 & 26,4 & 15,7 & 11,0 \\
\hline
\end{tabular}

Quellen: IPTS und OECD Directorate for Science, Technology and Innovation; EPO; eigene Berechnungen.

und Nordamerika ist etwa ausgeglichen. US-amerikanische Unternehmen forschen aber deutlich mehr in Asien als europäische Unternehmen.

Der größte Technologiebereich der Forschung ist die Elektrotechnik, auf die jeweils rund $45 \%$ der Patentgewichte sowohl insgesamt als auch im Ausland entfallen. Zwischen den einzelnen Technologiefeldern variiert der Internationalisierungsgrad deutlich weniger als zwischen den Heimatländern der Unternehmen. Im Ausland betreiben Unternehmen meist aus einer Position der heimischen technologischen Stärke FuE. Aber auch die Zielländer sind in den meisten Fällen auf genau diese Felder spezialisiert. Das Motiv der Internationalisierung ist dann der Erwerb von ergänzendem Wissen im Kompetenzfeld. Das zweitwichtigste Motiv ist die Nutzung des heimischen Wissensvorsprungs, um Produkte und Prozesse an Bedingungen im Ausland und an Kundenwünsche anzupassen. Weniger als $10 \%$ machen technologiesuchende Internationalisierungsstrategien aus, mit denen die Unternehmen versuchen, im Ausland ihre technologischen Defizite auszugleichen. Die gegenseitige Verflechtung zwischen den beiden großen Forschungsstandorten USA und Deutschland zeigt eine auffällige Ähnlichkeit der technologischen Schwerpunktsetzung der Unternehmen im jeweils anderen Land. Es dominieren in beide Richtungen wissensergänzende Internationalisierungsstrategien. Marktbezogene Motive haben US-Unternehmen in Deutschland vor allem in den Computer- und luK-Technologien, in denen sie klare Vorteile besitzen. Bei deutschen Unternehmen in den USA sind marktorientierte Motive vor allem in ihren Spezialisierungsfeldern elektrische Maschinen und Anlagen sowie Transporttechnik zu erkennen.

Insgesamt liefert unsere Studie keine Anhaltspunkte für die Befürchtung, dass FuE im Ausland zum Nachteil des Heimatstandorts durchgeführt wird. Aus Sicht der Unternehmen ist Auslandsforschung zur Sicherung der technologischen Wettbewerbsfähigkeit und zur Erweiterung ihres Absatzes notwendig. Da die wichtigsten Heimatländer der international forschenden Unternehmen gleichzeitig die wichtigsten Zielländer der FuE ausländischer Unternehmen sind, dürfte in den meisten Fällen eine Win-win-Situation bestehen. 
Abbildung 6

Internationalisierungsstrategien deutscher und US-Unternehmen nach Technologien (Patentgewichte)

US-Unternehmen in Deutschland

$0 \quad 100 \quad 200 \quad 300 \quad 400 \quad 500 \quad 600 \quad 700$

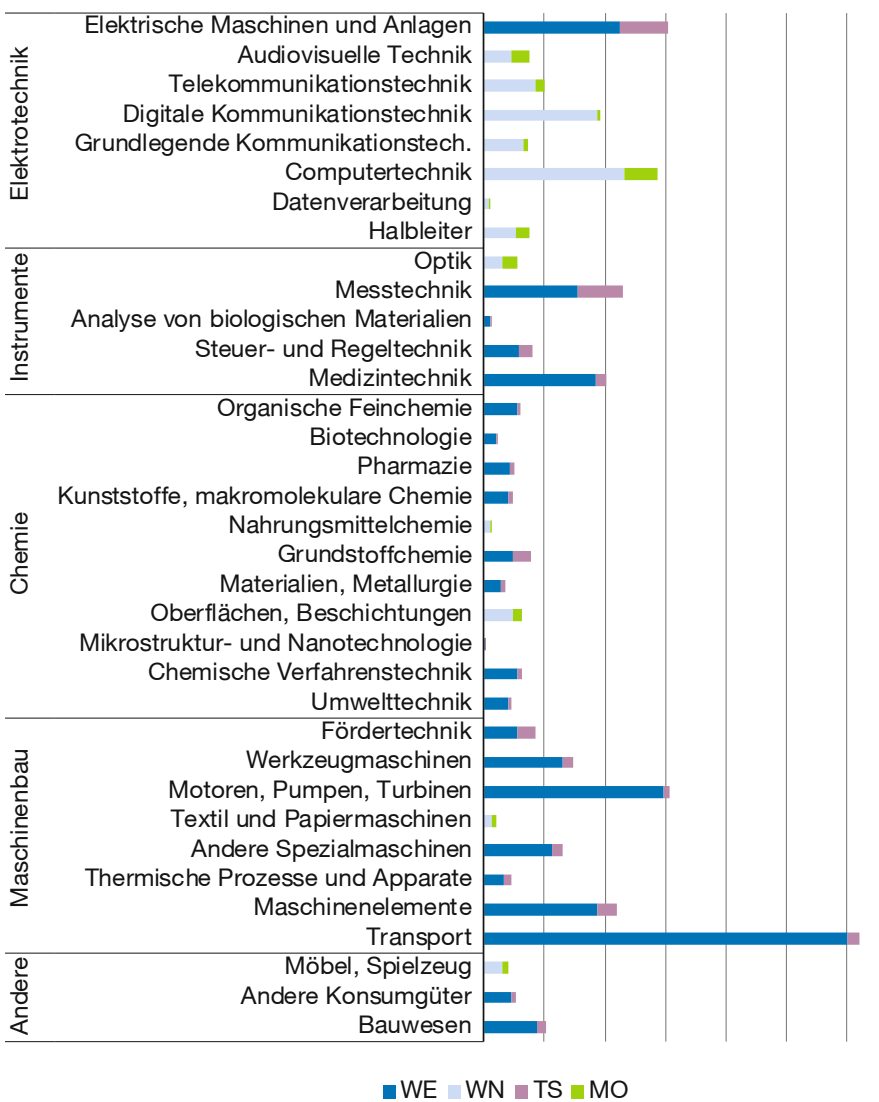

Deutsche Unternehmen in den USA

Quellen: IPTS und OECD Directorate for Science, Technology and Innovation; EPO; eigene Berechnungen.

\section{Literatur}

Belitz, H., A. Lejpras, A. Mattes und M. Priem (2019), Forschung deutscher Unternehmen im Ausland - Technologische Schwerpunkte und Zielregionen, Hans-Böckler-Stiftung, Working Paper Forschungsförderung Nr. 156, August 2019.

Gehrke, B., U. Schasse, H. Belitz, V. Eckl und G. Stenke (2020), Forschung und Entwicklung in Staat und Wirtschaft, Studien zum deutschen Innovationssystem, 2, Expertenkommission Forschung und Innovation.

Laurens, P., C. Le Bas, A. Schoen, L. Villard und P. Larédo (2015), The rate and motives of the internationalisation of large firm R\&D (1994-2005): Toward a turning point?, Research Policy, 44(3), 765-776.

Le Bas, C. und C. Sierra (2002), Location versus home country advantages in R\&D activities: some further results on multinationals' locational strategies, Research Policy, 31, 589-609. $\begin{array}{llllllll}0 & 100 & 200 & 300 & 400 & 500 & 600 & 700\end{array}$

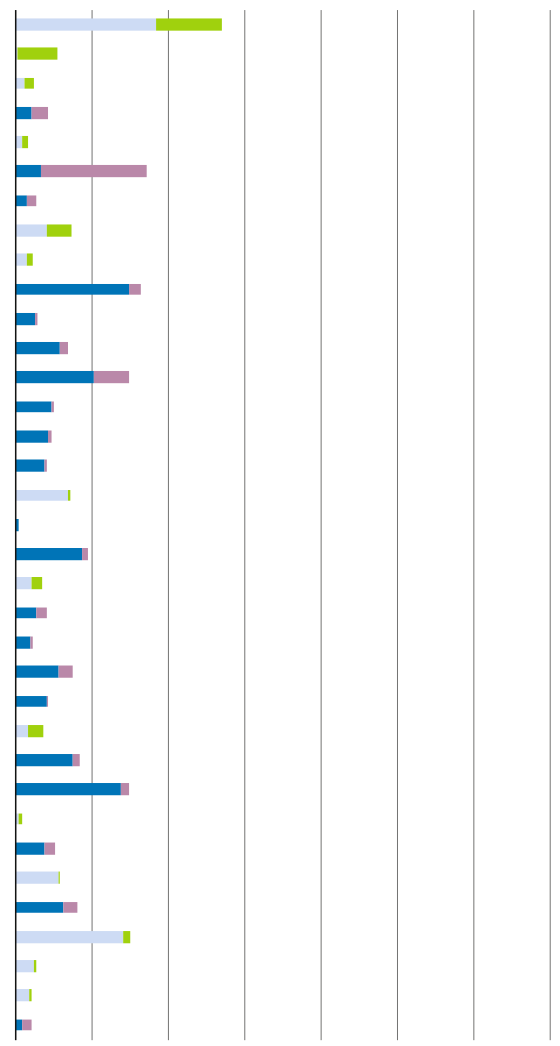

$-W E-W N-T S-M O$

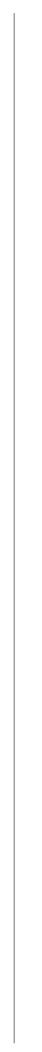

